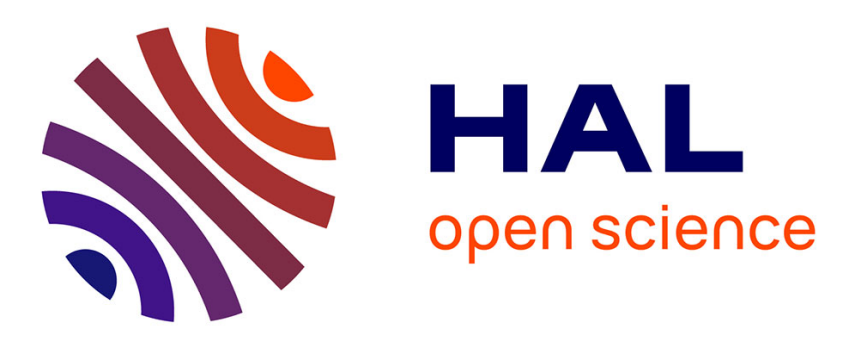

\title{
An Enhanced Information Structure for Linear Parameter-Varying Design: Application to Reichert's Missile Benchmark
}

Safta de Hillerin, Vincent Fromion, Gérard Scorletti, Gilles Duc, Emmanuel Godoy

\section{To cite this version:}

Safta de Hillerin, Vincent Fromion, Gérard Scorletti, Gilles Duc, Emmanuel Godoy. An Enhanced Information Structure for Linear Parameter-Varying Design: Application to Reichert's Missile Benchmark. AIAA Guidance, Navigation, and Control Conference, Aug 2010, Toronto, ON, Canada. 18 p., 10.2514/6.2010-8196 . hal-01676646

\section{HAL Id: hal-01676646 https://hal.science/hal-01676646}

Submitted on 5 Jan 2018

HAL is a multi-disciplinary open access archive for the deposit and dissemination of scientific research documents, whether they are published or not. The documents may come from teaching and research institutions in France or abroad, or from public or private research centers.
L'archive ouverte pluridisciplinaire HAL, est destinée au dépôt et à la diffusion de documents scientifiques de niveau recherche, publiés ou non, émanant des établissements d'enseignement et de recherche français ou étrangers, des laboratoires publics ou privés. 


\title{
An Enhanced Information Structure for Linear Parameter-Varying Design: Application to Reichert's Missile Benchmark
}

\author{
Safta de Hillerin ${ }^{1}$, Vincent Fromion ${ }^{2}$, Gérard Scorletti ${ }^{3}$, Gilles Duc ${ }^{4}$, and Emmanuel Godoy ${ }^{5}$ \\ ${ }^{1,4,5}$ SUPELEC Systems Sciences (E3S), Automatic Control Department, France \\ ${ }^{2}$ Institut National de la Recherche en Agronomie, Unité de Mathématique, Informatique et \\ Génome UR 1077, France \\ ${ }^{3}$ Ecole Centrale de Lyon, Laboratoire Ampère UMR CNRS 5005, France
}

This paper is concerned with the application of linear parameter-varying (LPV) methods. Its purpose is to investigate the interest of a new information structure for the LPV controllers.

The proposed improvement consists in extending the traditional information structure by introducing, beside the signals usually measured, special signals supposed available for control. This enhances the design in two directions: first, the performance of the obtained controller is improved by a more accurate adjustment to the LPV system parameter value; second, this structure enables the implementation of a controller of reduced complexity in relation to the LPV system parameter.

The advantages of the proposed structure are illustrated on the single-axis missile control problem proposed by Reichert which has been intensively studied in the existing literature.

\section{Introduction}

A linear parameter-varying (LPV) system is defined as:

$$
z=G_{L P V}(w)\left\{\begin{aligned}
\dot{x}(t) & =A(\theta(t)) x(t)+B(\theta(t)) w(t) \\
z(t) & =C(\theta(t)) x(t)+D(\theta(t)) w(t) \\
x\left(t_{0}\right) & =x_{0}
\end{aligned}\right.
$$

where $x(t) \in \mathbb{R}^{n}$ is the state vector, $w(t) \in \mathbb{R}^{n_{w}}$ is the disturbance input, $z(t) \in \mathbb{R}^{n_{z}}$ is the output and $\theta(t) \in \mathbb{R}^{p}$ is a time-varying exogenous parameter vector valued in a hypercube (each parameter $\theta_{i}(t)$ ranges between known extremal values $\underline{\theta}_{i}$ and $\bar{\theta}_{i}$ ).

The interest of these systems lies in the fact that they can model linear time-varying (LTV) and nonlinear plants. Assuming that the parameter is measured, the idea is to use the parameter measurements to improve the design compared to a linear time-invariant (LTI) strategy.

A customary method for finding a parameter-dependent controller (also denoted by gain-scheduled controller) was developed heuristically by engineers from LTI methods, see reference. ${ }^{1}$ It consists in designing LTI controllers using linearizations of the plant associated to frozen values of the parameter. The parameter-dependent controller is then obtained by interpolating these LTI controllers as functions of the parameter. Although this 
method is largely and quite successfully applied, it cannot a priori guarantee the obtained closed-loop performance and even stability, so that in practice engineers need to a posteriori test the performance by intensive dynamical simulations.

These serious drawbacks motivated the search for a systematic approach to construct a gain-scheduled controller, see e.g. references. ${ }^{1,2}$ Important contributions in this field are dated from the beginning of the 90's and are due to Packard. ${ }^{3}$ The LPV problem was formulated as the problem of minimizing the $\mathcal{L}_{2}$ gain of a system augmented with weighting functions, known as the $\mathcal{L}_{2}$ gain LPV control problem, which is an extension of the $H_{\infty}$ control problem. Indeed, an LTI plant is a very specific case of LPV plant and moreover, the $\mathcal{L}_{2}$ gain of an LTI system is equal to its $H_{\infty}$ norm so that in the case of an LTI plant, the $\mathcal{L}_{2}$ gain LPV control problem reduces to the $H_{\infty}$ control problem. The issue was then to obtain tractable conditions to solve the problem. The $\mathcal{L}_{2}$ gain LPV control problem turned out to be difficult: indeed, so far, in the general case only sufficient conditions could be written as a convex LMI optimization problem, hence tractable. These methods may therefore be over conservative. Numerous approaches were proposed. The simplest are based on quadratic Lyapunov functions and are therefore conservative in the case where parameters have bounded rates of variation: Packard ${ }^{3}$ or Apkarian and Gahinet ${ }^{4}$ recast the problem as a robust synthesis problem and solved it using a scaled version of the small-gain theorem, leading to convex conditions expressed as Linear Matrix Inequalities (LMIs). However, since the scaled small-gain theorem is only concerned with symmetric scalings, these procedures are unable to take into account the fact that the parameters are known to be real. Based on the exploitation of interconnected systems properties, less conservative results were obtained by Scorletti and El Ghaoui ${ }^{5}$ by introducing skew-symmetric scalings and by Scherer ${ }^{6}$ using full-block scalings.

Parameter-dependent Lyapunov functions can further reduce conservatism, however they lead to parameterdependent LMI optimization problems which are in general not tractable and methods for transforming these problems into tractable problems usually introduce conservatism, see e.g. references. ${ }^{7-10}$

Despite these theoretical limitations, in practise these methods yield encouraging results since it has become possible to obtain a controller that guarantees the closed-loop stability and performance. However, some points moderate these successes. Indeed, it was observed from the study of frozen linearizations that the obtained controller seems not to adjust much to the parameter value, see e.g. the conclusions in reference. ${ }^{11}$ Traditionally, this phenomenon was charged on the account of the conservatism introduced by the methods for solving the $\mathcal{L}_{2}$ gain LPV control problem. Another limitation of the LPV synthesis methods is that they produce controllers of high complexity in the parameter, thus possibly involving heavy computations for implementation.

The present investigation suggests another explanation for the phenomenon. Indeed, because LPV methods arise from LTI methods (and more precisely from the $H_{\infty}$ method), in engineering practise some processes that are usual and legitimate in an LTI framework have been transposed to the LPV context ad hoc, that is, sometimes without further investigation on the validity of the analogy. In particular, the adequacy of the traditional LTI information structure (that is, the choice of signals available for control) in the LPV context has not really been investigated.

This paper focuses on the issue of the selection of control signals and suggests a seemingly more suitable choice: the idea is to introduce for control, besides the classical measures, two other available signals.

One signal is a system output that gives information about the operating point. This leads to significant amelioration of the design, as will be demonstrated by comparison with classical results: first, it ameliorates the performance level and second, the frozen linearizations indicate that the controller adjusts better to the parameter variations.

The other signal introduced in order to further enhance the design is the signal corresponding to the parameter-dependent term in the state-space equations, which can be supposed available without making fur- 
ther hypotheses. The idea for this structure was first introduced by $\mathrm{Wu}$ and $\mathrm{Lu}^{12}$ and a contribution of the present paper is to suggest a way to exploit that idea to improve the design of LPV controllers. The result in reference $^{12}$ implies indeed that the new structure is all the more interesting that incidentally it enables to obtain a controller of reduced complexity in the parameter, hence involving less computations and thus circumventing a major limitation of the usual synthesis methods.

The paper is organized as follows. The missile model and the design objectives of the benchmark proposed by Reichert are presented in Section II. An LPV controller is calculated in Section III using a classical information structure. A new information structure is proposed in Section IV, where it is shown that the resulting LPV controller has a better dependence on the parameter and therefore achieves better performance. In Section V, it is proved that the structure presents moreover the practical interest of permitting the implementation of a controller of reduced complexity in the parameter. Concluding remarks end the paper in Section VI.

\section{Notations}

The notation is fairly standard. $M^{T}$ is the transpose of matrix $M$. For a symmetric real matrix $M, M>0$ and $M<0$ stand respectively for positive definite and negative definite while $M \geq 0$ and $M \leq 0$ stand respectively for nonnegative and nonpositive definite. The Laplace variable is denoted by $s$ and $\dot{x}=\frac{d x}{d t}$ is the time derivative. $I_{n}$ is used to denote the identity matrix of size $n$ and $O_{m \times n}$ the zero matrix of dimensions $m \times n$ but when dimensions are obvious from context, only the notation $I$ and $O$ may be used. The maximal and minimal singular values of a matrix $M$ are denoted respectively by $\bar{\sigma}(M)$ and $\underline{\sigma}(M)$. The state-space realization of transfer $G(s)=D+C(s I-A)^{-1} B$ is denoted by $G(s)=\left[\begin{array}{l|l}A & B \\ \hline C & D\end{array}\right]$. The $H_{\infty}$ norm of a stable LTI system $G$ with transfer function $G(s)$ is denoted by $\|G\|_{\infty}$ and defined as $\|G\|_{\infty}=\sup _{\omega \in[0,+\infty)} \bar{\sigma}(G(j \omega))$.

Next are given some definitions and notations specific to the LPV context. We introduce the augmented LPV plant $P_{L P V}$ :

$$
\left[\begin{array}{l}
z \\
y
\end{array}\right]=P_{L P V}\left[\begin{array}{l}
w \\
u
\end{array}\right]\left\{\begin{aligned}
\dot{x}(t) & =A(\theta(t)) x(t)+B_{w}(\theta(t)) w(t)+B_{u}(\theta(t)) u(t) \\
z(t) & =C_{z}(\theta(t)) x(t)+D_{z w}(\theta(t)) w(t)+D_{z u}(\theta(t)) u(t) \\
y(t) & =C_{y}(\theta(t)) x(t)+D_{y w}(\theta(t)) w(t) \\
x\left(t_{0}\right) & =x_{0}
\end{aligned}\right.
$$

where $u(t) \in \mathbb{R}^{n_{u}}$ is the controlled input and $y(t) \in \mathbb{R}^{n_{y}}$ is the measured output. In the proposed approaches of references, ${ }^{3,4,13}$ the dependence on the parameters of the state-space matrices is supposed to be rational. The methods then require the LPV plant $P_{L P V}$ to be written as the interconnection of an LTI plant $P(s)$ with a so-called parameter block matrix $\Theta$ characterizing the parameter structure. This is called the linear fractional transform (LFT) representation. For matrices $\Theta=\left[\begin{array}{l|l}\Theta_{11} & \Theta_{12} \\ \hline \Theta_{21} & \Theta_{22}\end{array}\right]$ and $M$ of compatible dimensions, $\mathcal{F}_{l}(M, \Theta)=\Theta_{11}+\Theta_{12} M\left(I-\Theta_{22} M\right)^{-1} \Theta_{21}$ denotes the lower LFT of the interconnection $(M, \Theta)$ and $\mathcal{F}_{u}(M, \Theta)=\Theta_{22}+\Theta_{21} M\left(I-\Theta_{11} M\right)^{-1} \Theta_{12}$ the upper LFT. In this paper, recall that the parameter vector is defined as $\theta=\left[\theta_{1} \cdots \theta_{p}\right]^{T}$ and is assumed to be real. The parameter block is then defined as a diagonal matrix $\Theta=\operatorname{diag}\left(\theta_{1} I_{n_{1}}, \ldots, \theta_{p} I_{n_{p}}\right)$ where $n_{i}$ is the number of times $\theta_{i}$ appears in the LFT. The dimension (or size) of the parameter block is then $n_{\theta}=n_{1}+\cdot+n_{p}$.

In the approaches considered, the LPV controller $K_{L P V}$ is assumed to have the same dependency on the parameter as the plant, therefore it is also written in LFT form as the interconnection of an LTI system $K(s)$ and the same parameter block $\Theta$ as the plant. Notice that the closed-loop system from $w$ to $z$ represented in 
Figure 1 denoted by $P_{L P V} \star K_{L P V}$ reads in LFT form: $\mathcal{F}_{l}\left(\mathcal{F}_{u}(P(s), \theta), \mathcal{F}_{l}(K(s), \theta)\right)$.

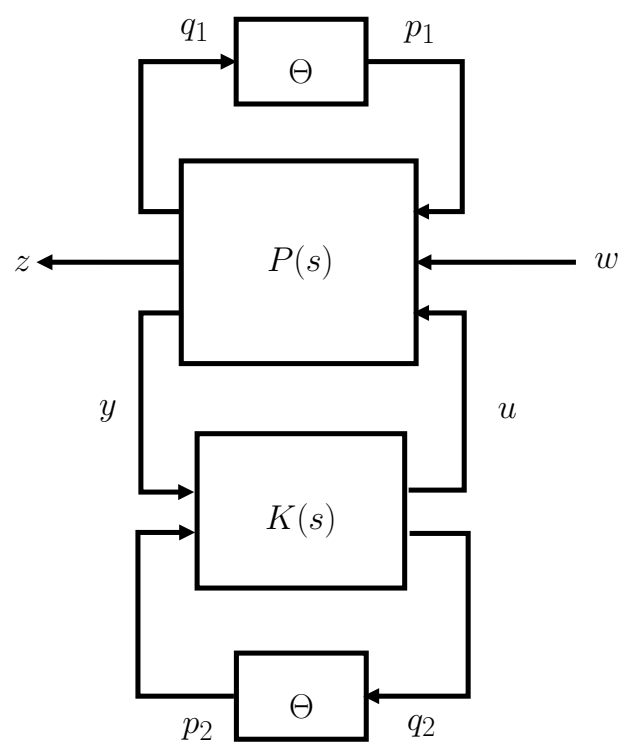

Figure 1. Closed-loop LPV system in LFT representation.

The $\mathcal{L}_{2}$ gain of an LPV system $z=G_{L P V}(w)$ defined as in (1) is the smallest $\gamma$ such that for all $T_{0} \geq t_{0}$, we have

$$
\int_{t_{0}}^{T_{0}} z(t)^{T} z(t) d t \leq \gamma^{2} \int_{t_{0}}^{T_{0}} w(t)^{T} w(t) d t
$$

for any $w$ such that $\int_{t_{0}}^{T_{0}} w(t)^{T} w(t) d t<\infty$.

For an LPV augmented plant $P_{L P V}$ defined as in (2), the $\mathcal{L}_{2}$ gain LPV control problem can be stated as follows: Design an LPV controller $u=K_{L P V}(y)$ such that, with the closed loop system represented Figure 1 and defined by $P_{L P V} \star K_{L P V}=\mathcal{F}_{l}\left(\mathcal{F}_{u}(P(s), \theta), \mathcal{F}_{l}(K(s), \theta)\right)$ :

- $P_{L P V} \star K_{L P V}$ is asymptotically stable;

- $P_{L P V} \star K_{L P V}$ has a $\mathcal{L}_{2}$ gain less than a given $\gamma \geq 0$ (known as level of performance).

Tractable sufficient conditions for this problem were derived by Apkarian and Gahinet ${ }^{4}$ as an LMI feasibility problem. All the results presented in the next Sections were obtained by implementing the formulae in reference ${ }^{4}$ which are given in detail in the appendix at Section VII.

\section{Model of the missile and design specifications}

\section{A. Nonlinear model of the missile}

The considered system is the pitch-axis model of a missile, flying at Mach 3 and at an altitude of 20, $000 \mathrm{ft}$, that was defined by Reichert. ${ }^{14}$ The associated control problem was intensively studied, see e.g. references. ${ }^{11,15-19}$

The idea is to use the tail deflection $\delta$ to track an acceleration maneuver. The missile is modeled as a rigid body, see Figure 2. The control input is $\delta$ and the measured outputs are the acceleration $\eta$ and the pitch rate $q$. The state of the missile involves the angle of attack $\alpha$ and the pitch rate $q$ and the state-space equations are:

$$
\left\{\begin{aligned}
\dot{\alpha} & =\cos (\alpha) K_{\alpha} M C_{n}(\alpha, \delta, M)+q \\
\dot{q} & =K_{q} M^{2} C_{m}(\alpha, \delta, M)
\end{aligned}\right.
$$


The acceleration output $\eta$ is given by:

$$
\eta=\frac{K_{z}}{g} M^{2} C_{n}(\alpha, \delta, M)
$$

where $M$ is the Mach while the functions $C_{n}$ and $C_{m}$ are defined by:

$$
\left\{\begin{aligned}
C_{n}(\alpha, \delta, M) & =a_{n} \alpha^{3}+b_{n}|\alpha| \alpha+c_{n}(2-M / 3) \alpha+d_{n} \delta \\
C_{m}(\alpha, \delta, M) & =a_{m} \alpha^{3}+b_{m}|\alpha| \alpha+c_{m}(-7+8 M / 3) \alpha+d_{m} \delta
\end{aligned}\right.
$$

For this specific model, these two functions are determined. However in practice, the coefficients are usually known only poorly and sometimes not at all. It is interesting to emphasize that the approach described below can be applied even in these cases.

The actuator is modeled as a second order system:

$$
\ddot{\delta}=-\omega_{a}^{2} \delta-2 \xi_{a} \omega_{a} \dot{\delta}+\omega_{a}^{2} \delta_{c}
$$

where $\delta$ is the actual tail deflection and $\delta_{c}$ the commanded tail deflection.

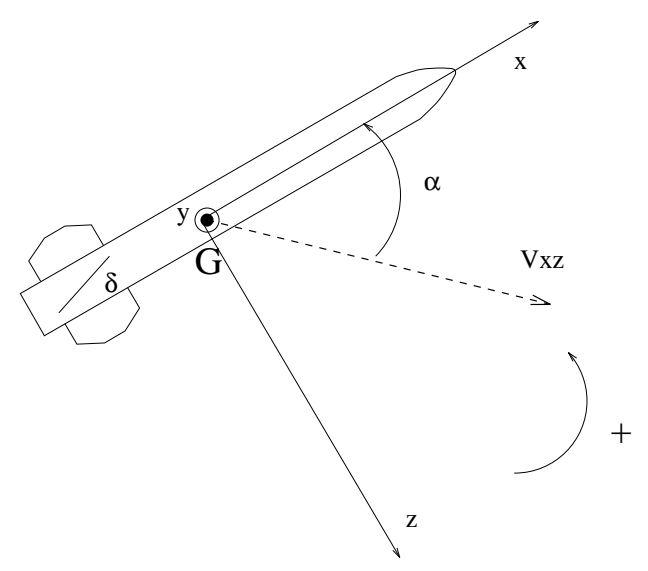

Figure 2. Definition of the missile variables.

See Table 1 for the associated numerical data extracted from Reichert's paper. ${ }^{14}$

\section{B. Design objectives}

The design specifications considered follow from Ferreres et al. ${ }^{16}$ and were used as well in references: ${ }^{11,20}$

- when applying a step input signal to the reference input $\eta_{c}(t)$, the time constant must be less than $0.35 s$, the maximal overshoot less than $20 \%$ and the steady state error less than $5 \%$;

- actuator saturation, both in acceleration and in speed, should be avoided;

- due to the presence of non modeled flexible modes, the controller bandwidth must be limited (the transfer from $\eta_{c}$ to $\eta$ must present an attenuation of $30 \mathrm{~dB}$ at $300 \mathrm{rad} / \mathrm{s}$ );

- robustness to uncertainties on aerodynamic functions coefficients is demanded.

\section{LPV model of the missile}

In order to apply LPV synthesis methods to this system, the first task is to derive an LPV model from the nonlinear model of the missile and to write it in LFT form. Following the lines of reference, ${ }^{20}$ we use the 


\begin{tabular}{|c|cc|}
\hline$a_{n}$ & $1.028610^{-4}$ & $\mathrm{deg}^{-3}$ \\
\hline$b_{n}$ & $-0.9445710^{-2}$ & $\mathrm{deg}^{-2}$ \\
\hline$c_{n}$ & -0.1696 & $\mathrm{deg}^{-1}$ \\
\hline$d_{n}$ & -0.034 & $\mathrm{deg}^{-1}$ \\
\hline$a_{m}$ & $2.152410^{-4}$ & $\mathrm{deg}^{-3}$ \\
\hline$b_{m}$ & $-1.954610^{-2}$ & $\mathrm{deg}^{-2}$ \\
\hline$c_{m}$ & 0.051 & $\mathrm{deg}^{-1}$ \\
\hline$d_{m}$ & -0.206 & $\mathrm{deg}^{-1}$ \\
\hline$\omega_{a}$ & 150 & \\
\hline$\xi_{a}$ & 0.7 \\
\hline
\end{tabular}

\begin{tabular}{|c|c|}
\hline$P_{0}$ & $973.3 \mathrm{lb} / \mathrm{ft}^{2}$ \\
\hline$S$ & $0.44 \mathrm{ft}^{2}$ \\
\hline$m$ & 13.98 slugs \\
\hline$V$ & $1036.4 \mathrm{ft} / \mathrm{s}$ \\
\hline$d$ & $0.75 \mathrm{ft}$ \\
\hline$I_{y}$ & $182.5 \mathrm{slug} . \mathrm{ft}$ \\
\hline$K_{\alpha}$ & $0.7 \mathrm{PoS} / \mathrm{m} / \mathrm{V}$ \\
\hline$K_{q}$ & $0.7 \mathrm{PoSd} / I_{y}$ \\
\hline$K_{z}$ & $0.7 \mathrm{PoS} / \mathrm{m}$ \\
\hline$g$ & $\begin{array}{r}32.2 \\
\hline\end{array}$ \\
\hline
\end{tabular}

Table 1. Missile parameters.

approximations $\cos (\alpha) \approx 1$ for the considered angles. Two polytopic models are introduced: one corresponds to the original nonlinear model written in quasi-LPV form, that is, considering the nonlinear dependence as embedded in a parameter. The other corresponds to the non stationary linearizations of the model. Since $a_{m}=2 a_{n}, b_{m}=2 b_{n}$, the equations obtained in these two cases are the same, the difference lying in the definition of the parameter: thus, if we wish to consider the nonlinear system then $\theta(t)=a_{m} \alpha(t)^{3}+b_{m} \alpha(t)^{2}$, whereas if we consider the non stationary linearizations in $\alpha_{0}$ we should take $\theta(t)=3 a_{m} \alpha_{0}(t)^{2}+2 b_{m}\left|\alpha_{0}(t)\right|$. Since the angle $\alpha$ varies between $-0.35 \mathrm{rad}$ and $0.35 \mathrm{rad}, a_{m} \alpha^{3}+b_{m} \alpha^{2}$ varies between 0 and -10 while $3 a_{m} \alpha^{2}+2 b_{m}|\alpha|$ varies between 0 and -15 . Hence by considering that the parameter varies between 0 and -15 we take into account both the nonlinear model and its non stationary linearizations.

The state-space equations are given below, where the state is $x=[\alpha q]^{T}$ :

$$
\left[\begin{array}{c}
\dot{x} \\
\eta
\end{array}\right]=\left[\begin{array}{ll}
A+\theta(t) A_{\theta} & B \\
C+\theta(t) C_{\theta} & D
\end{array}\right]\left[\begin{array}{l}
x \\
\delta
\end{array}\right]
$$

where

$$
\begin{aligned}
{\left[\begin{array}{l|l}
A & B \\
\hline C & D
\end{array}\right]=} & {\left[\begin{array}{cc|c}
K_{\alpha} M c_{n}\left(2-\frac{M}{3}\right) & 1 & K_{\alpha} M d_{n} \\
K_{q} M^{2} c_{m}\left(-7+\frac{8}{3} M\right) & 0 & K_{q} M^{2} d_{m} \\
\hline \frac{K_{z}}{g} M^{2} c_{n}\left(2-\frac{M}{3}\right) & 0 & \frac{K_{z}}{g} M^{2} d_{n}
\end{array}\right] } \\
& {\left[\begin{array}{c}
A_{\theta} \\
\hline C_{\theta}
\end{array}\right]=\left[\begin{array}{cc}
K_{\alpha} M & 0 \\
2 K_{q} M^{2} & 0 \\
\hline \frac{K_{z}}{g} M^{2} & 0
\end{array}\right] . }
\end{aligned}
$$

The equations can be further written in LFT form by isolating the parameter-dependent signal. Thus in the LFT representation, the parameter block input is defined as $q_{1}(t)=\alpha(t)$ and the parameter block output is 
$p_{1}(t)=\theta(t) \cdot q_{1}(t)$. Then the state-space equations become the following:

$$
\begin{gathered}
{\left[\begin{array}{c}
\dot{x} \\
\hline q_{1} \\
\eta
\end{array}\right]=\left[\begin{array}{c|cc}
A & A_{\theta} & B \\
\hline\left[\begin{array}{cc}
1 & 0
\end{array}\right] & 0 & 0 \\
C & C_{\theta} & D
\end{array}\right]\left[\begin{array}{c}
x \\
\hline p_{1} \\
\delta
\end{array}\right] .} \\
p_{1}(t)=\theta(t) \cdot q_{1}(t)
\end{gathered}
$$

Notice that in this particular example there is a single parameter so that the parameter block $\Theta$ depicted in Figure 1 and later in Figure 11 is in fact a scalar and therefore will usually be denoted by $\theta$.

\section{Control with the classical information structure}

We first review the results obtained with an information structure that was classically considered in literature, where the controller inputs are the pitch rate $q$ and the tracking error $\eta_{c}-\eta$. More specifically, the information structure considered is directly inspired from references. ${ }^{11,16}$

\section{A. Criterion and weighting functions for the classical information structure}

We proceed as for an usual $H_{\infty}$ synthesis, see e.g. reference: ${ }^{21}$ the performance specifications are characterized by LTI weighting functions constraining the closed-loop transfer functions. Thus following the lines of reference, ${ }^{16}$ we use the 6 blocs criterion described in Figure 3 to specify performance and robustness.

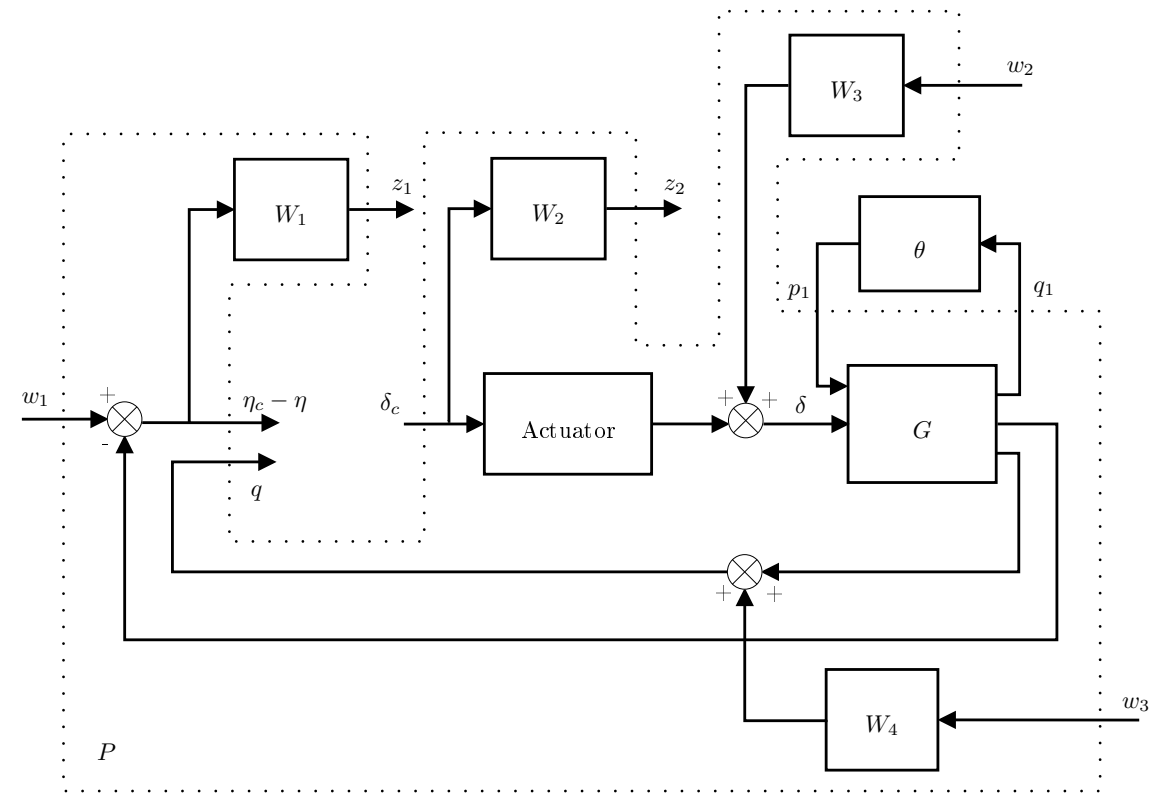

Figure 3. $H_{\infty}$ criterion for the classical information structure (controller inputs: $\eta_{c}-\eta$ and $q$ ).

To choose adequate weighting functions, it is in general necessary to do several trials, see reference. ${ }^{21}$ The usual method goes as follows: first, weights are sought for one special plant LTI frozen linearization like for an usual $H_{\infty}$ synthesis procedure. Next, the functions are modified until they are suitable for the plant LTI frozen linearizations corresponding to all the parameter values in the definition set. A satisfying result was obtained in reference ${ }^{11}$ with the weighting functions given below. The corresponding frequency responses for $W_{1}(s)$ and 
$W_{2}(s)$ are displayed in Figure 4.

$$
W_{1}(s)=10^{3} \frac{s / 6.93+1}{s / 3.46 \cdot 10^{-3}+1}, \quad W_{2}(s)=10 \frac{s^{2} / 150^{2}+0.8 / 150 s+1}{s^{2} / 1000^{2}+2 / 1000 s+1}, \quad W_{3}(s)=0.04, \quad W_{4}(s)=0.07 .
$$

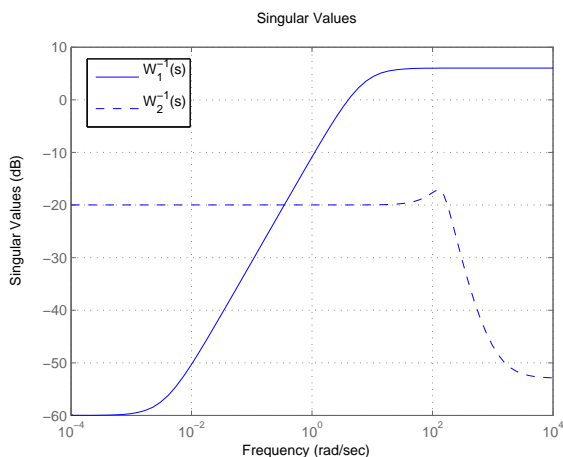

Figure 4. Weighting functions $W_{1}(s)$ and $W_{2}(s)$.

\section{B. Results for the classical information structure}

The obtained level of performance is $\gamma=1.297$. The results that were obtained by performing an LPV synthesis with the classical information structure of Figure 5 are illustrated in Figure 5 (LTI frozen linearizations) and Figure 6 (LPV simulations for the parameter trajectory corresponding to the nonlinear (quasi-LPV) model $\theta(t)=a_{m} \alpha(t)^{3}+b_{m} \alpha(t)^{2}$ for several step inputs of different amplitudes).

The results obtained by performing an LPV synthesis using the classical structure are quite satisfying: the LTI and LPV plots enable indeed to check that the design specifications are respected (time response about $3 \mathrm{~s}$ and overshoot less than 20\%). Moreover, contrary to the usual heuristic gain-scheduled methods, LPV methods guarantee that the stability and performance properties are achieved for any parameter trajectory remaining in the definition set.

A question nevertheless arises from the study of the LTI plots. Theoretically, we expect here to obtain a parameter-dependent controller. However, the frequency responses of the controller LTI frozen linearizations seem to indicate that the influence of the parameter is weak. This is confirmed by the fact that the frequency responses of the closed-loop LTI frozen linearizations still depend on the parameter. As an undesirable consequence, the step responses of the LTI frozen linearizations and of the LPV simulations of the controlled system are not homogeneous enough $\left(e . g . t_{5 \%} \in[0.3,0.5] s\right)$.

Having recalled these classical results, we next propose a modified information structure that leads to better results.

\section{Control with the new information structure}

First, a new structure is presented and the interest of the signal newly introduced is justified empirically. An LPV synthesis is then carried out while the weighting functions are kept the same as previously, in order to highlight the potential by comparing the level of performance obtained. Next, the structure is further modified by introducing another signal supposed available, which presents another interest.

Next, the weights are modified accurately to improve the performance. An LPV synthesis is carried out and a comparative study of the LTI frozen plots and the LPV simulations is presented, emphasizing the advantages of the new structure. 

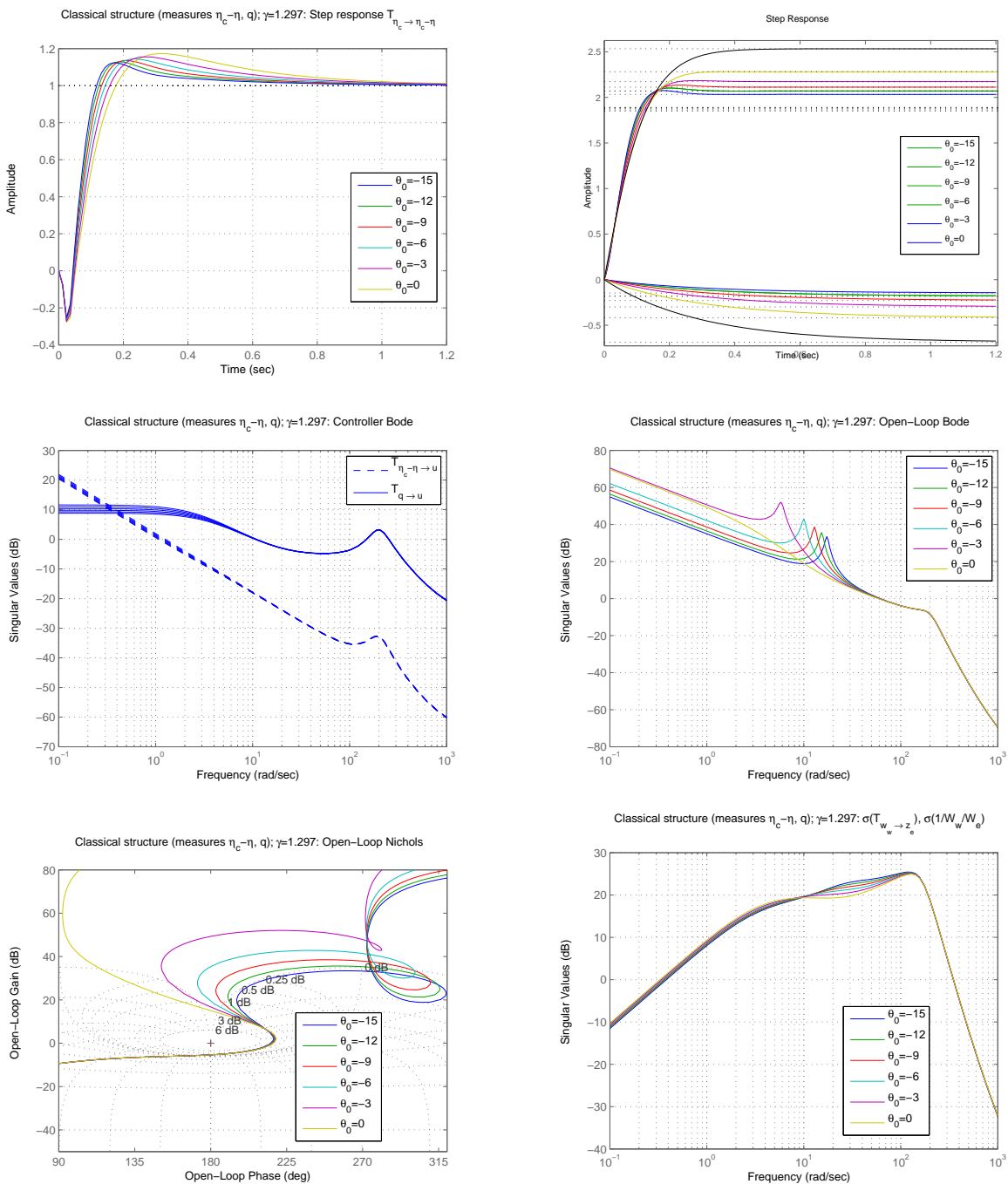

Figure 5. Frozen LTI plots for the classical information structure (controller inputs: $\eta_{c}-\eta$ and $q$ ): Step response from $\eta_{c}$ to $\eta(1)$, Closed-loop step response of dominant poles from $\eta_{c}$ to $\eta$ (2), Controller Bode (3), Open-loop Bode (4), Open-loop Black-Nichols (5), Closed-loop Bode from $w_{3}$ to $\left(\eta_{c}-\eta\right)(6)$.
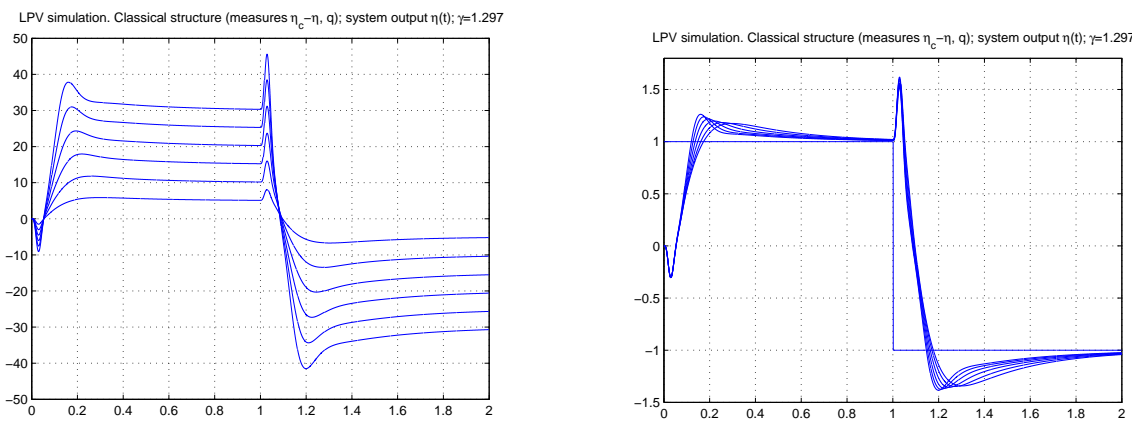

Figure 6. LPV simulation for the classical information structure (controller inputs: $\eta_{c}-\eta$ and $q$ ) with $\theta(t)=$ $a_{m} \alpha(t)^{3}+b_{m} \alpha(t)^{2}$ : Step response $\eta(t)$ for different step sizes (1) and brought to the same scale (2). 


\section{A. Criterion and weighting functions for the new information structure}

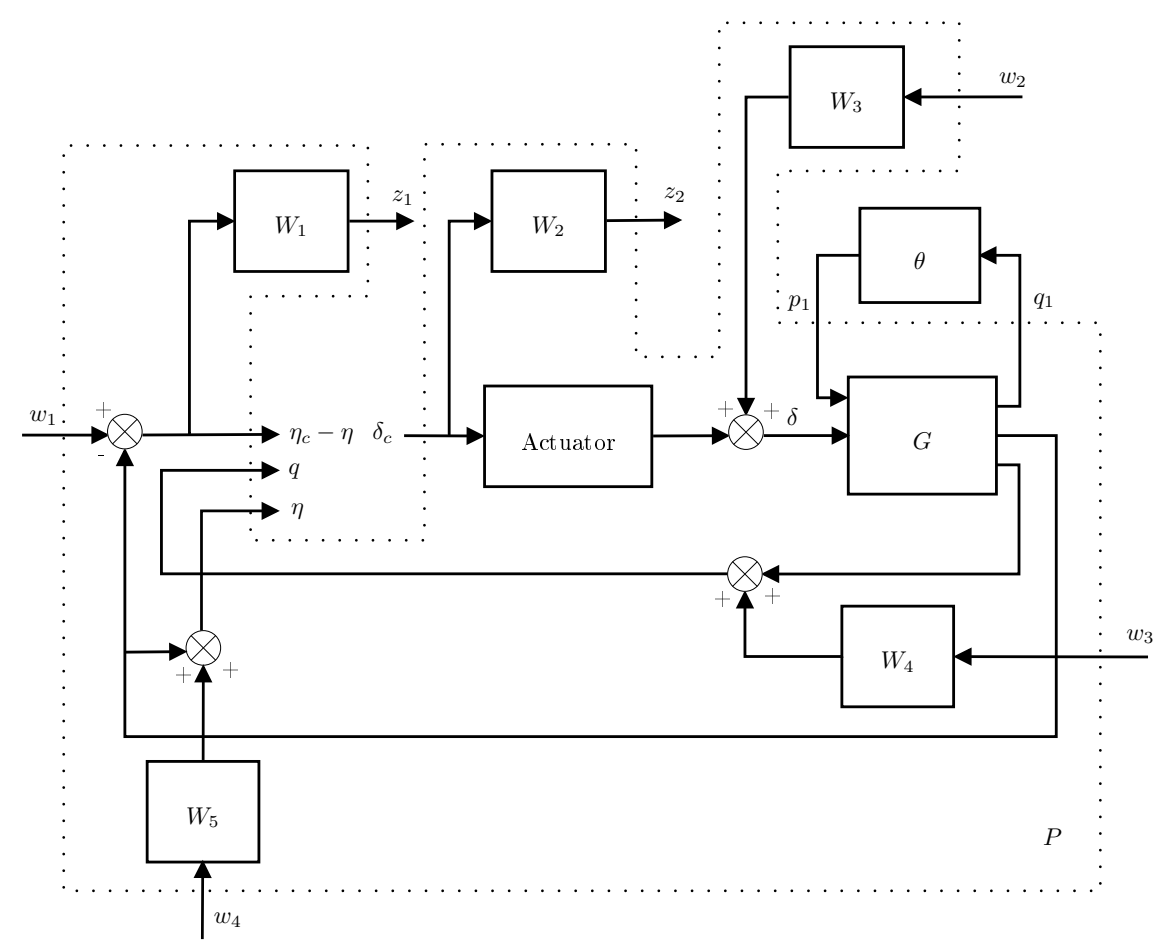

Figure 7. $H_{\infty}$ criterion for the new information structure (controller inputs: $\eta_{c}-\eta, q$ and $\eta$ ).

The new control criterion considered is given in Figure 7. The difference with the classical structure lies in the fact that here besides the tracking error and the pitch rate, the acceleration $\eta$ is explicitly fed back as a controller input. It is clear that by adding an extra measure the level of performance will be at least as good as previously, however this is not the only reason why we suggest adding this signal. Indeed, in the classical structure, the fact that only the tracking error is measured implies that potentially crucial information about the operating point is lacking. Therefore, by using $\eta$ not only better performance is expected but also a controller that can adjust better to the parameter value.

Using the same weighting functions as for the usual information structure (see Section III), we obtain with this new structure a performance level $\gamma=1.19$. This suggests that the performance can be further improved by an adequate choice of the weighting functions. However, we are not yet interested in this issue at this stage. Rather, we seek to further improve the design by adding another signal that also proves useful: it is the output $\theta(t) \cdot q_{1}(t)$ of the parameter block in the LFT representation of the plant.

In the specific case of the considered missile model it corresponds to the parameter-dependent term in the plant state-space equations. We do not make a strong assumption by supposing that this signal is available for control: indeed, to design an LPV controller of the missile it is already assumed that the parameter $\theta(t)$ is available in real time. However, recall that $\theta(t)$ is a polynomial in $\alpha(t)$ and that in the LPV model, the system output $q_{1}(t)$ is simply $\alpha(t)$. Therefore, supposing that the output of the parameter block (which is here simply the parameter-dependent term) is available for control is a "realistic hypothesis". The new structure considered is depicted in Figure 8. Performing an LPV synthesis leads to a level of performance $\gamma=1.18$ (which is not much different than the level obtained with only the measures $\left.\eta_{c}-\eta, q, \eta\right)$. In order to enable an accurate comparison with the classical structure, the weighting function $W_{1}$ is adjusted so that the time response is 


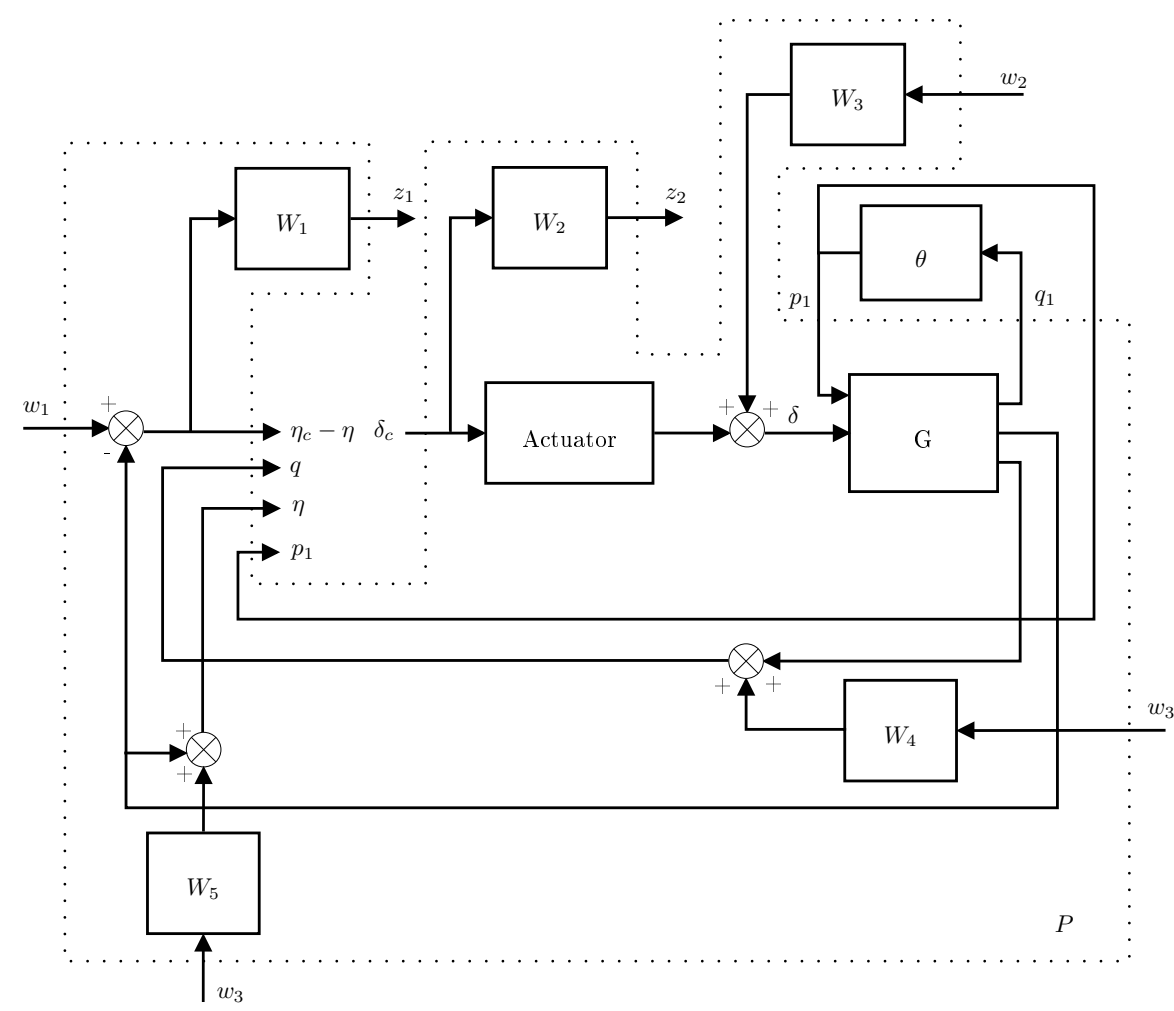

Figure 8. $H_{\infty}$ criterion for the new information structure (controller inputs: $\eta_{c}-\eta, q, \eta$ and $\theta \cdot \alpha$ ).

smaller:

$$
W_{1}(s)=\frac{5.5 \cdot 10^{-1} s+8.35}{s+8.4 \cdot 10^{-3}} .
$$

\section{B. Results for the new information structure}

By performing an LPV synthesis with the new information structure of Figure 8 and the adjusted weighting functions, the level of performance $\gamma=1.288$ is reached. Illustrating plots are displayed in Figure 9 (LTI frozen linearizations) and Figure 10 (LPV simulations for the parameter trajectory corresponding to the nonlinear (quasi-LPV) model $\theta(t)=a_{m} \alpha(t)^{3}+b_{m} \alpha(t)^{2}$ for several step inputs of different amplitudes).

The plots point out that significant improvement is achieved with the new structure. The performance is obviously ameliorated, as highlighted by the fact that while the margins and the level of performance remain the same, the controlled system follows the reference input much better: from both LTI and LPV plots, it is clear that the step responses are quicker and more homogeneous (e.g. on the LTI plots, $\left.t_{5 \%} \in[0.2,0.3] s\right)$. This is consistent with the LTI Bode plots of the controller indicating that it adjusts much better to the parameter value.

\section{A practical advantage of the new structure}

This section focuses on an interesting practical advantage of the new structure from the implementation point of view. Actually, the fact that the term $\theta(t) \cdot \alpha(t)$ is supposed available enables to construct a controller of reduced complexity in the parameter. This interesting property is the consequence of a theorem established by $\mathrm{Wu}$ and Lu. ${ }^{12}$

The interpretation of the theorem which is made here is nevertheless quite different: reference ${ }^{12}$ is actually 

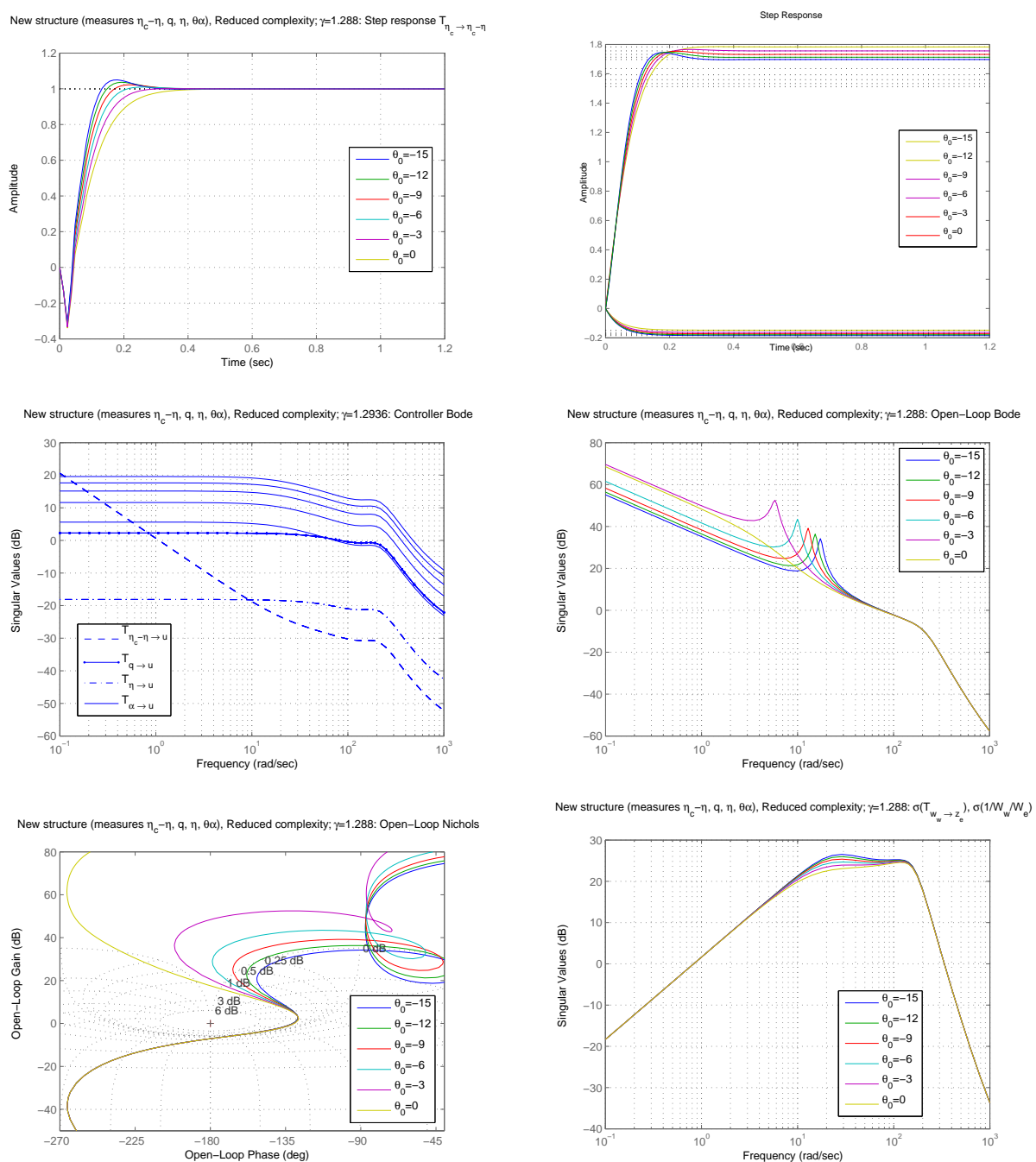

Figure 9. Frozen LTI plots for the new information structure (controller inputs: $\eta_{c}-\eta, q, \eta$ and $\theta \cdot \alpha$ ): Step response from $\eta_{c}$ to $\eta(1)$, Closed-loop step response of dominant poles from $\eta_{c}$ to $\eta$ (2), Controller Bode (3), Open-loop Bode (4), Open-loop Black-Nichols (5), Closed-loop Bode from $w_{3}$ to $\left(\eta_{c}-\eta\right)(6)$.
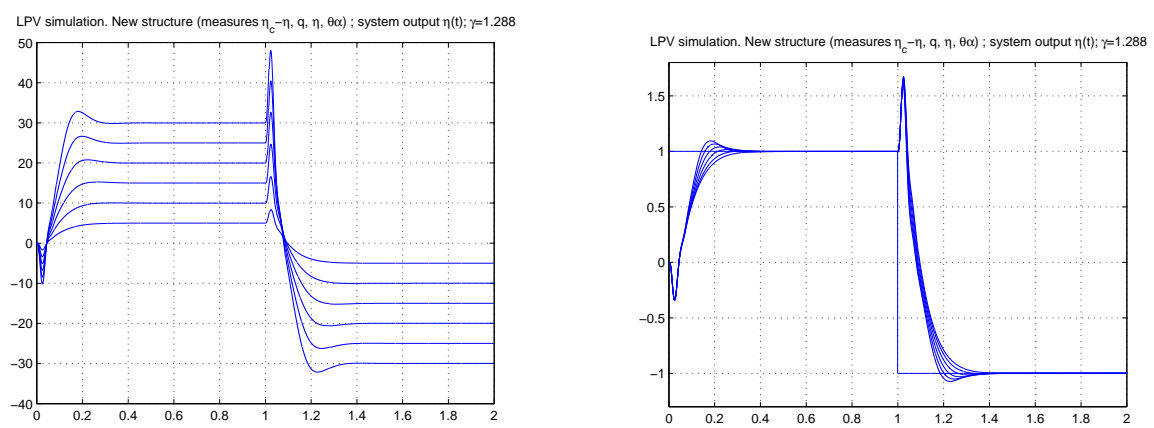

Figure 10. LPV simulation for the new information structure (controller inputs: $\eta_{c}-\eta, q, \eta$ and $\theta \cdot \alpha$ ) with $\theta(t)=a_{m} \alpha(t)^{3}+b_{m} \alpha(t)^{2}$ : Step response $\eta(t)$ for different step sizes (1) and brought to the same scale (2). 
concerned with conditions for LTI robust synthesis. These are in the general case nonconvex ones, see $e . g$. reference. ${ }^{22}$ More precisely, in reference, ${ }^{12}$ the conditions considered are the ones introduced by Apkarian and Gahinet. ${ }^{4}$ Yet the theorem implies that if the special signal corresponding to the output of the parameter block of the plant is available for control, then the conditions for LTI robust synthesis are simplified: they turn into an LMI feasibility problem, thus becoming convex. In fact, it is proved that in presence of this particular measure, the (generally nonconvex) LTI robust synthesis problem becomes equivalent to the (convex) $\mathcal{L}_{2}$ gain LPV control problem.

In our context, the theorem implies a very interesting result if it is interpreted in the following manner: if the output of the parameter block of the plant is available for control, then the conditions of the $\mathcal{L}_{2}$ gain LPV control problem are equivalent to the conditions of the robust LTI control problem. This means that if the $\mathcal{L}_{2}$ gain LPV control problem is solvable, then it is possible to construct a controller that has an LTI structure, as depicted in Figure 11. It is important to notice that it does not make it an LTI controller in the usual sense because here one input of the controller is a parameter-varying signal (since it is the output of the plant parameter block i.e. the signal $\left.\theta(t) \cdot q_{1}(t)\right)$. Equivalently, this can be summarized by saying that if the parameter-dependent signal is available for control, then it is possible to construct an LPV controller whose LFT block parameter dimension is zero. A sketch of the proof of this theorem is provided in Section VII.

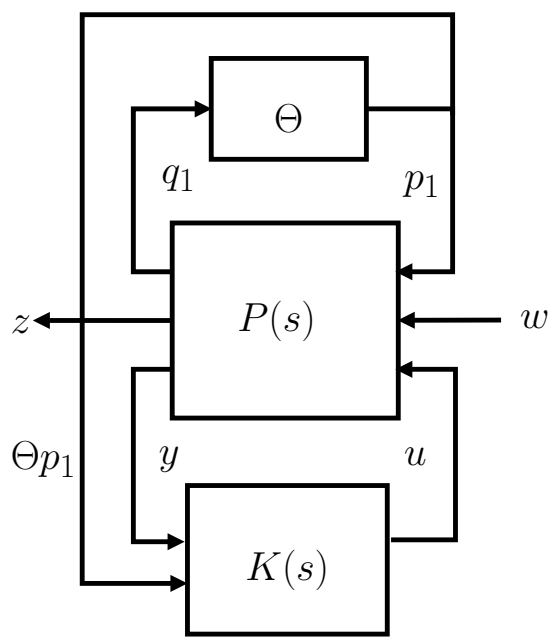

Figure 11. Closed-loop LPV system with controller of reduced complexity in the parameter.

This property has a great advantage from a practical point of view. Indeed, the controller complexity is one of the main limitations of the implementation of LPV methods. Rewriting the system equations in a lower fractional manner often leads to defining a parameter block of great dimension $n_{\theta}$. While an usual LPV synthesis would lead to a controller having the same complexity as the plant (that is, having a parameter block of dimension $n_{\theta}$ ), the new structure enables to construct a controller having a parameter block of dimension zero.

Here the controller can be constructed as:

$$
u=\left[\begin{array}{llll}
K_{\left(\eta_{c}-\eta\right) \rightarrow u}(s) & K_{q \rightarrow u}(s) & K_{\eta \rightarrow u}(s) & K_{(\theta \cdot \alpha) \rightarrow u}(s)
\end{array}\right]\left[\begin{array}{c}
\eta_{c}-\eta \\
q \\
\eta \\
\theta \cdot \alpha
\end{array}\right] .
$$


The structure of the controller and its dependence on the parameter in thus clear: the controller is LTI with an input that is parameter-dependent. The Bode plots of to each transfer function are given separately in Figure 12 (see also Figure $9(3))$.
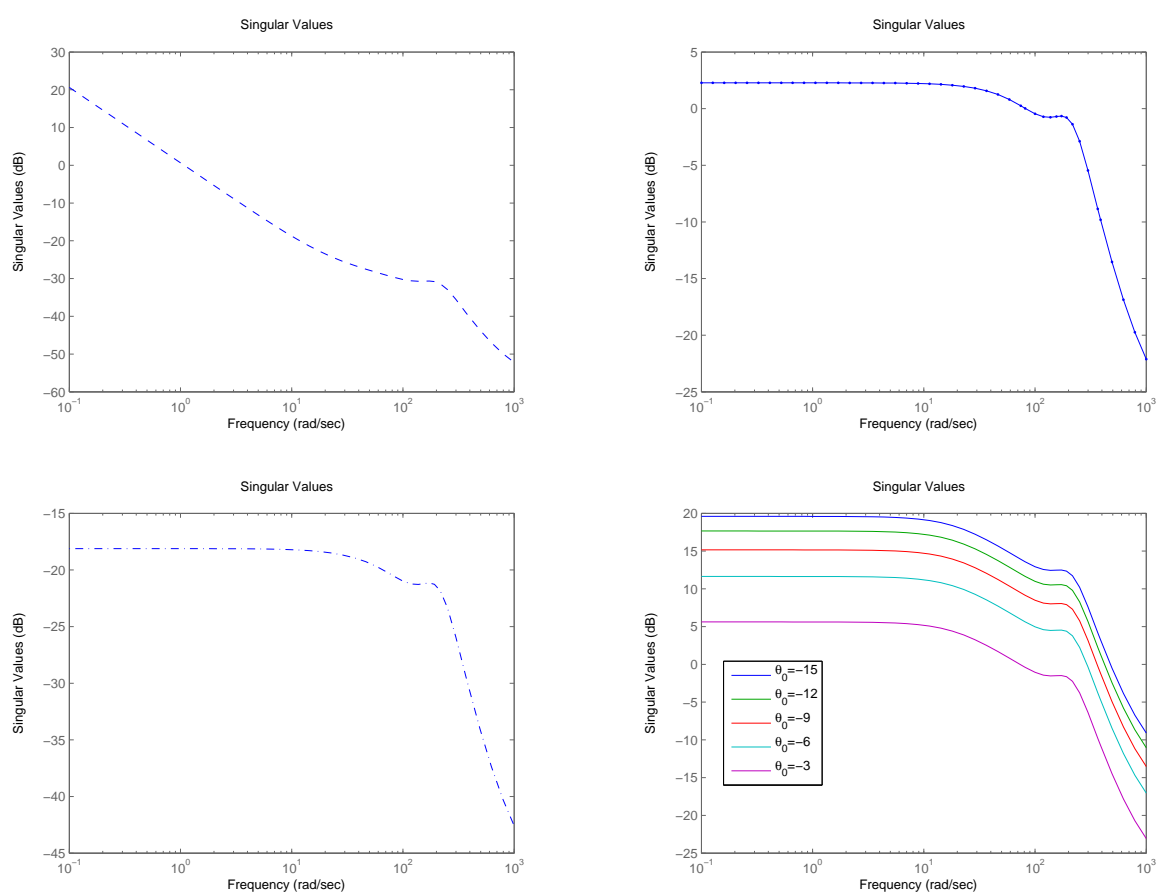

Figure 12. Frozen LTI plots of the controller: $K_{\left(\eta_{c}-\eta\right) \rightarrow u}(1), K_{q \rightarrow u}(2), K_{\eta \rightarrow u}(3), \theta \cdot K_{\alpha \rightarrow u}(4)$.

A simplified expression of the transfer functions (after model reduction by truncation to the order 4 ) is given below:

$$
\left\{\begin{array}{l}
K_{\left(\eta_{c}-\eta\right) \rightarrow u}(s)=2.65 \cdot 10^{-3} \frac{(s+38.03)(s+19.82)\left(s^{2}-1.48 \cdot 10^{2} s+2 \cdot 10^{6}\right)}{\left(s+7.79 \cdot 10^{2}\right)\left(s+1.95 \cdot 10^{2}\right)(s+24.46)\left(s+8.15 \cdot 10^{-3}\right)} \\
K_{q \rightarrow u}(s)=0.15 \frac{(s+27.17)\left(s+8.16 \cdot 10^{-3}\right)\left(s^{2}-2.96 \cdot 10^{2} s+1.17 \cdot 10^{6}\right)}{\left(s+7.79 \cdot 10^{2}\right)\left(s+1.95 \cdot 10^{2}\right)(s+24.46)\left(s+8.15 \cdot 10^{-3}\right)} \\
K_{\eta \rightarrow u}(s)=2.15 \cdot 10^{-2} \frac{(s-26.56)\left(s+8.15 \cdot 10^{-3}\right)\left(s^{2}-3.38 \cdot 10^{2} s+8.10 \cdot 10^{6}\right)}{\left(s+7.79 \cdot 10^{2}\right)\left(s+1.95 \cdot 10^{2}\right)(s+24.46)\left(s+8.15 \cdot 10^{-3}\right)} \\
K_{(\theta \cdot \alpha) \rightarrow u}(s)=-7.30 \cdot 10^{-2} \frac{(s-43.1)\left(s+8.15 \cdot 10^{-3}\right)\left(s^{2}-3.35 \cdot 10^{2} s+7.53 \cdot 10^{5}\right)}{\left(s+7.79 \cdot 10^{2}\right)\left(s+1.95 \cdot 10^{2}\right)(s+24.46)\left(s+8.15 \cdot 10^{-3}\right)}
\end{array}\right.
$$

\section{Conclusions}

In this paper, the interest of a new information structure for LPV synthesis is investigated and illustrated on the Reichert's missile control problem. The proposed improvement consists in augmenting the classical structure by supposing that besides the usually considered measures of the acceleration tracking error and the pitch rate, two other signals are used for control: first, the actual acceleration and second, the parameter-dependent signal. It is shown on this demonstrative example that the new structure yields improved performance and leads to a controller that adjusts better to the parameter value. Furthermore, it enables to construct a controller that has reduced complexity in relation to the parameter.

The proposed solution supposes nevertheless that the measure of the parameter block output $\theta(t) \cdot q_{1}(t)$ is 
directly available. A more realistic hypothesis would be that this signal is not perfectly measured or, worse, has to be estimated. Robustness of the proposed solution to uncertainties on the measure of the parameter block output $\theta(t) \cdot q_{1}(t)$ is under investigation.

\section{Appendix: Draft of proof of the reduction of the controller complexity in the parameter}

This section proposes a sketch of an alternative proof of the interesting result established by Wu and Lu. ${ }^{12}$ The proof somehow differs from that in reference ${ }^{12}$ as the result is interpreted here in the context of LPV control. Recall indeed that in reference, ${ }^{12}$ the aim was to show that the presence of the plant parameter block output enables a relaxation of the nonconvex LTI robust control conditions, which then become convex and in fact equivalent to the conditions of the $\mathcal{L}_{2}$ gain LPV control problem.

Here the base is the classical LPV problem considered $e . g$. in references. ${ }^{3-6}$ The proof goes as follows: first are recalled the LMI conditions for the $\mathcal{L}_{2}$ gain LPV control problem as they are introduced by Apkarian and Gahinet. ${ }^{4}$ Next it is shown that these conditions are simplified if the plant parameter block output is available for control, thus yielding some freedom in the decision variables. These variables can then be chosen such that the complexity of the controller in relation to the parameter is reduced, that is, more precisely, such that the parameter block in the LFT representation of the controller is of dimension zero.

Denote by $P$ the system formed by the original plant augmented with the weighting functions of Figure 3 . The augmented system matrices are defined in LFT form as follows:

$$
\left[\begin{array}{c}
\dot{x} \\
q_{1} \\
z \\
y
\end{array}\right]=\left[\begin{array}{cccc}
A & B_{\theta} & B_{1} & B_{2} \\
C_{\theta} & D_{\theta \theta} & D_{\theta 1} & D_{\theta 2} \\
C_{1} & D_{1 \theta} & D_{11} & D_{12} \\
C_{2} & D_{2 \theta} & D_{21} & 0
\end{array}\right]\left[\begin{array}{c}
x \\
p_{1} \\
w \\
u
\end{array}\right] .
$$

Sufficient conditions for the $\mathcal{L}_{2}$ gain LPV control problem are obtained by Apkarian and Gahinet ${ }^{4}$ as the following LMI feasibility problem: Find, if they exist, symmetric positive definite matrices $R, S \in \mathbb{R}^{n \times n}$, 
$J_{3}, L_{3} \in \mathbb{R}^{n_{\theta} \times n_{\theta}}$ satisfying the LMIs (10), (11), (12), (13):

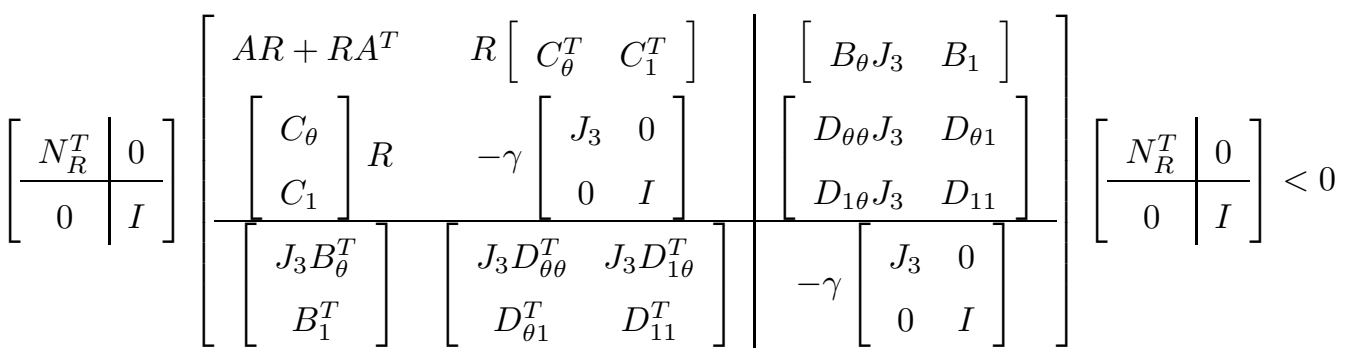

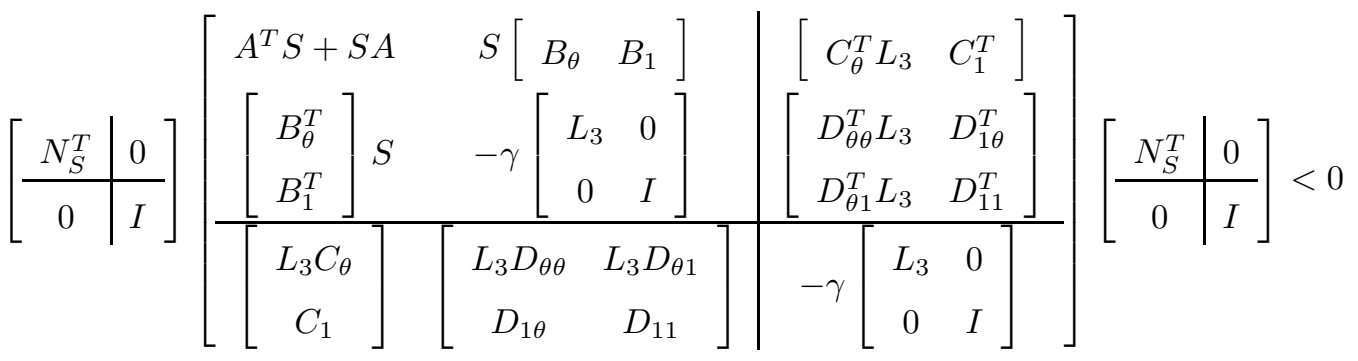

$$
\begin{gathered}
{\left[\begin{array}{cc}
R & I \\
I & S
\end{array}\right] \geq 0} \\
{\left[\begin{array}{cc}
L_{3} & I \\
I & J_{3}
\end{array}\right] \geq 0}
\end{gathered}
$$

with $N_{R}=\operatorname{Ker}\left(\left[\begin{array}{ccc}B_{2}^{T} & D_{\theta 2}^{T} & D_{12}^{T}\end{array}\right]\right), N_{S}=\operatorname{Ker}\left(\left[\begin{array}{lll}C_{2} & D_{2 \theta} & D_{21}\end{array}\right]\right)$.

Recall that the rank $k$ of matrix $I-R S$ defines the number of states of the controller while the rank $r$ of the matrix $I-L_{3} J_{3}$ defines the dimension of the parameter block in the LFT representation of the controller.

In the special case where the output of the parameter block is measured as in Figure 8 , the matrices $C_{2}, D_{2 \theta}$ and $D_{21}$ can be partitioned so that:

$$
\left[\begin{array}{cccc}
C_{2} & D_{2 \theta} & D_{21} & 0
\end{array}\right]=\left[\begin{array}{cccc}
\hat{C}_{2} & \hat{D}_{2 \theta} & \hat{D}_{21} & 0 \\
0 & I & 0 & 0
\end{array}\right]
$$

Consequently, one can write $N_{S}=\left[\begin{array}{cc}W_{1} & 0 \\ 0 & 0 \\ W_{3} & 0 \\ 0 & I\end{array}\right]$ where the matrices $W_{1}$ and $W_{3}$ are such that $\hat{N}_{S}=\left[\begin{array}{c}W_{1} \\ W_{3}\end{array}\right] \in \operatorname{Ker}\left(\left[\begin{array}{c}\hat{C}_{2} \\ \hat{D}_{21}\end{array}\right]\right)$ and $\left[\begin{array}{cc}\hat{C}_{2}^{T} & W_{1} \\ \hat{D}_{21}^{T} & W_{3}\end{array}\right]$ is full-rank.

After rewriting the LMI (11) and applying the elimination lemma (see reference ${ }^{23}$ ) followed by the Schur lemma to eliminate the decision variable $L_{3}$, the LMI feasibility problem ((10), (11), (12), (13)) in $\left(R, S, J_{3}, L_{3}\right)$ becomes the LMI feasibility problem $((14),(15),(16),(17))$ in $\left(R, S, J_{3}\right)$ : 


$$
\begin{aligned}
& N_{R}^{T}\left[\begin{array}{ccc}
A R+R A^{T} & R\left[\begin{array}{cc}
C_{\theta}^{T} & C_{1}^{T}
\end{array}\right] & {\left[\begin{array}{cc}
B_{\theta} J_{3} & B_{1}
\end{array}\right]} \\
{\left[\begin{array}{c}
C_{\theta} \\
C_{1}
\end{array}\right] R} & -\gamma\left[\begin{array}{cc}
J_{3} & 0 \\
0 & I
\end{array}\right] & {\left[\begin{array}{cc}
D_{\theta \theta} J_{3} & D_{\theta 1} \\
D_{1 \theta} J_{3} & D_{11}
\end{array}\right]} \\
{\left[\begin{array}{c}
J_{3} B_{\theta}^{T} \\
B_{1}^{T}
\end{array}\right]} & {\left[\begin{array}{cc}
J_{3} D_{\theta \theta}^{T} & J_{3} D_{1 \theta}^{T} \\
D_{\theta 1}^{T} & D_{11}^{T}
\end{array}\right]} & -\gamma\left[\begin{array}{cc}
J_{3} & 0 \\
0 & I
\end{array}\right]
\end{array}\right] N_{R}<0 \\
& \hat{N}_{S}^{T}\left[\begin{array}{ccc}
A^{T} S+S A & S B_{1} & {\left[\begin{array}{cc}
C_{\theta}^{T} & C_{1}^{T}
\end{array}\right]} \\
B_{1}^{T} S & -\gamma I & {\left[\begin{array}{ll}
D_{\theta 1}^{T} & D_{11}^{T}
\end{array}\right]} \\
{\left[\begin{array}{c}
C_{\theta} \\
C_{1}
\end{array}\right]} & {\left[\begin{array}{c}
D_{\theta 1} \\
D_{11}
\end{array}\right]} & -\gamma\left[\begin{array}{cc}
J_{3} & 0 \\
0 & I
\end{array}\right]
\end{array}\right] \hat{N}_{S}<0 \\
& {\left[\begin{array}{ll}
R & I \\
I & S
\end{array}\right] \geq 0} \\
& J_{3} \geq 0
\end{aligned}
$$

where $N_{R}=\operatorname{Ker}\left(\left[\begin{array}{cccc}B_{2}^{T} & D_{\theta 2}^{T} & D_{12}^{T} & 0\end{array}\right]\right), \hat{N}_{S}=\operatorname{Ker}\left(\left[\begin{array}{lll}\hat{C}_{2} & \hat{D}_{21} & 0\end{array}\right]\right)$.

The elimination lemma implies that if there exists a solution $\left(R, S, J_{3}\right)$ of the LMI feasibility problem ((14), (15), (16), (17)), then there exists $L_{3}$ such that $\left(R, S, J_{3}, L_{3}\right)$ is a solution of the LMI feasibility problem ((10), (11), (12), (13)).

The next step is to notice that $L_{3}=J_{3}^{-1}$ is a suitable choice. Recalling that the dimension of the parameter block in the LFT representation of the controller is defined as the rank of matrix $I-L_{3} J_{3}$ concludes the proof.

\section{References}

${ }^{1}$ Rugh, W. J. and Shamma, J. S., "Research on Gain Scheduling," Automatica, Vol. 36, 2000, pp. 1401-1425.

${ }^{2}$ Fromion, V. and Scorletti, G., "A theoretical framework for gain scheduling," International Journal of Robust and Nonlinear Control, Vol. 13, Feb. 2003, pp. 951-982.

${ }^{3}$ Packard, A., "Gain Scheduling via Linear Fractional Transformations," Systems and Control Letters, Vol. 22, No. 2, Feb. 1994, pp. 79-92.

${ }^{4}$ Apkarian, P. and Gahinet, P., "A convex characterization of gain-scheduled $H_{\infty}$ controllers," IEEE Trans. Automatic Control, Vol. 40, No. 5, May 1995, pp. 853-864.

${ }^{5}$ Scorletti, G. and El Ghaoui, L., "Improved LMI Conditions for Gain Scheduling and Related Problems," International Journal of Robust and Nonlinear Control, Vol. 8, No. 10, Aug. 1998, pp. 845-877.

${ }^{6}$ Scherer, C. W., "LPV control and full block multipliers," Automatica, Vol. 37, 2001, pp. 361-375.

${ }^{7}$ Köse, I. E. and Jabbari, F., "Control of LPV systems with partly measured parameters," IEEE Trans. Automatic Control, Vol. 44, March 1999, pp. 658-663.

${ }^{8}$ Iwasaki, T. and Shibata, G., "LPV system analysis via quadratic separator for uncertain implicit systems," IEEE Trans. Automatic Control, Vol. 46, Aug. 2001, pp. 1195-1208.

${ }^{9}$ Bliman, P.-A., "Stabilization of LPV systems," Proc. IEEE Conf. on Decision and Control, Dec., pp. 6103-6108.

${ }^{10}$ M. Dinh, G. Scorletti, V. F. and Magarotto, E., "Parameter dependent $H_{\infty}$ control by finite dimensional LMI optimization: application to trade-off dependent control," International Journal of Robust and Nonlinear Control, Vol. 15, 2005, pp. 383-406.

${ }^{11}$ Scorletti, G., Approche unifiée de l'analyse et la commande des systèmes par formulation LMI, Ph.D. thesis, Université d'Orsay, Paris, France, 1997.

${ }^{12}$ Wu, F. and Lu, B., "On convexified robust control synthesis," Automatica, Vol. 40, 2004, pp. 1003 - 1010. 
${ }^{13}$ Scorletti, G., "A more praticle formulation for robustness analysis," IFAC Conference on System Structure and Control, Nantes, France, July 1998.

${ }^{14}$ Reichert, R. T., "Dynamic Scheduling of Modern-Robust-Control Autopilot Design for Missiles," IEEE Control Syst. Mag., Oct. 1992, pp. 35-42.

${ }^{15}$ Apkarian, P., Biannic, J., and Gahinet, P., "Self-Scheduled $H_{\infty}$ Control of Missile via Linear Matrix Inequalities," $A I A A$ J. Guidance, Control and Dynamics, Vol. 18, No. 3, May 1995, pp. 532-538.

${ }^{16}$ Ferreres, G., Fromion, V., Duc, G., and M'Saad, M., "Application of real/mixed $\mu$ computational techniques to an $H_{\infty}$ missile autopilot," International Journal of Robust and Nonlinear Control, Vol. 6, 1996, pp. 743-769.

${ }^{17}$ Nichols, R. A., Reichert, R. T., and Rugh, W. J., "Gain Scheduling for $H_{\infty}$ Controllers: A Flight Control Example," IEEE Trans. Control Sys. Tech., Vol. 1, No. 2, 1993, pp. 69-69.

${ }^{18}$ Shamma, J. S. and Cloutier, J. R., "Gain-Scheduled Missile Autopilot Design Using Linear Parameter Varying Transformation," AIAA J. Guidance, Control and Dynamics, Vol. 16, No. 2, March-April 1993, pp. 256-263.

${ }^{19}$ Balas, G. J., Lind, R., and Packard, A., "Optimal Scaled $H_{\infty}$ Full Information Control Synthesis with Real Uncertainty," AIAA J. Guidance, Control and Dynamics, Vol. 19, No. 4, July 1996, pp. 854-862.

${ }^{20}$ Fromion, V., Scorletti, G., and Ferreres, G., "Nonlinear performance of a PI controlled missile: an explanation," Proc. IEEE Conf. on Decision and Control, Vol. 9, June 1999.

${ }^{21}$ Skogestad, S. and Postlethwaite, I., Multivariable feedback control analysis and design, Wiley, 1996.

${ }^{22}$ Rotea, M. A. and Iwasaki, T., “An alternative to the D-K iteration,” Proc. American Control Conf., June 1994 , pp. 53-57.

${ }^{23}$ Gahinet, P. and Apkarian, P., "A Linear Matrix Inequality Approach to $H_{\infty}$ Control," International Journal of Robust and Nonlinear Control, Vol. 4, 1994, pp. 421-448. 